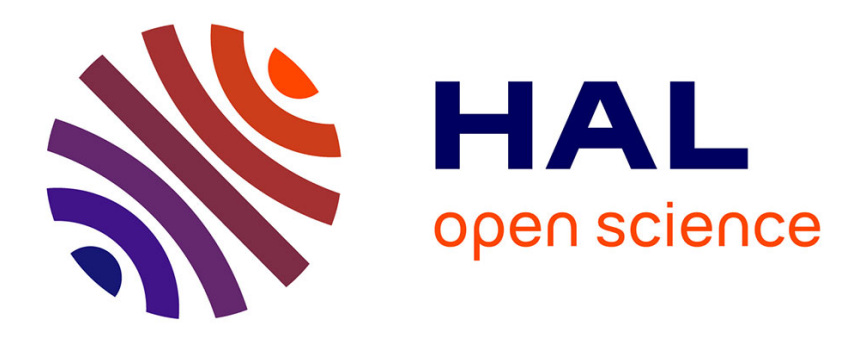

\title{
Temporal characterization of experimental recorder attack transients
}

Augustin Ernoult, Benoît Fabre

\section{To cite this version:}

Augustin Ernoult, Benoît Fabre. Temporal characterization of experimental recorder attack transients. Journal of the Acoustical Society of America, 2017, 141 (1), pp.383 - 394. 10.1121/1.4973861 . hal01468377

\section{HAL Id: hal-01468377 \\ https: / hal.sorbonne-universite.fr/hal-01468377}

Submitted on 15 Feb 2017

HAL is a multi-disciplinary open access archive for the deposit and dissemination of scientific research documents, whether they are published or not. The documents may come from teaching and research institutions in France or abroad, or from public or private research centers.
L'archive ouverte pluridisciplinaire HAL, est destinée au dépôt et à la diffusion de documents scientifiques de niveau recherche, publiés ou non, émanant des établissements d'enseignement et de recherche français ou étrangers, des laboratoires publics ou privés. 


\title{
Temporal characterization of experimental recorder attack transients.
}

\author{
A. Ernoult ${ }^{(*)} 1$, B. Fabre ${ }^{(*)}$ \\ (*) Sorbonne Universités, UPMC Univ Paris 06, CNRS, UMR 7190, LAM, Institut Jean Le \\ Rond $\partial$ 'Alembert, F-75005 Paris, France
}

\begin{abstract}
Through the comparison of notes produced on two recorders by one novice and two experienced players in musical contexts, this study identifies the temporal aspects of the attack transients which are controlled by the player and those which are imposed by the dynamic response of the instrument. The rise times of mouth pressure used by the novice player are longer than the ones produced by the experienced players, which are the shortest ones preserving the control of the jet velocity rise times. For shortest rise times, the jet development is limited by the inertia of the channel. The onset time of the acoustic oscillation is divided in two parts: the time needed to initiate the oscillation and the duration of the growth. The times of growth are similar for the novice and the experienced players, which is linked to the similarity of the gain characterizing the linear behavior of the instrument. The times needed to initiate the oscillation show the most pronounced difference between the two types of players; it is much longer for a novice player. This difference is interpreted in terms of the initial input of energy in the system which is larger for the experienced players.
\end{abstract}

Keywords: musical instruments, flute-like instruments, attack transients, instrumental control

PACS: 43.75Ef, 43.75Qr

\footnotetext{
${ }^{1}$ ernoult@lam.jussieu.fr
} 


\section{Introduction}

Playing music using self-sustained oscillations allows the player to shape the sound by modulating the control parameters. In recorder playing, the musician gives each note or group of notes a specific time evolution of the acoustic oscillation, according to the musical context and intention. Therefore, the sound properties, for a specific fingering, are always changing depending on the continuous adjustments of the musicians control parameters.

In this work, the attack transient is considered to be the transition between no sound and a stable oscillation. It starts when the musician starts to blow, a moment before the onset of sound production, and finishes when the amplitude and the frequency of the acoustic oscillation and the mouth pressure of the musician are quasi-steady. The attack transients are known to be very important for the perception of sound [1,2]. It contributes to the characterization of the timbre of instruments and the musicians adjust the transients according to the musical expression. Understanding the phenomena involved and the roles of the different parameters in the attack transients is important for instrument makers and for players.

The recorder is a flute-like instrument in which the sound is created by a jet-edge interaction coupled to a resonator. Some experimental works have already been carried out on the attack transients of this type of instrument, especially the organ pipe (Keeler [3], Nolle [4], Verge [5] , Castellengo [6]). It appears that the typical duration of an attack transient is about 10 to $30 \mathrm{~ms}$. It corresponds to a quick modification of the supply pressure in comparison to the acoustic period ( $2 \mathrm{~ms}$ for a oscillation at $500 \mathrm{~Hz})$. These studies present limited data and are focused on specific aspects of the transient. Since the instruments used in the different studies are different, they are not easily comparable.

The most common model used for flute-like instruments is the jet-drive source model (Coltman [7]). This model assumes that the jet is split in two by the edge. This split can be described as a dipole of flux placed on both sides of the edge. The model allows us to understand most of the stationary or quasi-static behaviors of flute-like instruments [8]. The jet-drive model has also been used to study the regime change in these instruments (Terrien [9]). However, as it assumes a well-defined jet, it can't a priori be used to study the attack transients. Indeed, there is no jet when the musician starts to blow. Some authors already proposed other models, sometimes with comparisons to experimental studies (Fletcher [10], Nolle [4], Verge [11]). The models are not necessarily linked to an acoustical understanding of transients (parameters are not linked with physical parameters, etc.). Additionally, only specific points of the attack transients is modeled.

Before developing a model, it is important to characterize the attack transients. The aim of this first study is to explore the characteristics of attack transients in recorder playing, varied by the musicians in a musical context, and to understand how the musicians control these characteristics.

Measurements are carried out on a family of recorders played by two experienced players and a novice player. The experienced players are expected to have good control of the 
instrument which allow them to consistently produce acceptable musical sounds. It is therefore sometimes hard to know if one aspect of the transient is due to this fine control by the musician or imposed by the instrument, as illustrated in a preliminary study [12]. The novice player is assumed to have a very poor control over the instrument, especially of the attack transient. The comparison of the attacks obtained by these two types of players should allow us to distinguish which characteristics of attack transients are controlled by the musician and which ones are imposed by the dynamic response of the instrument. Due to the difference of the behaviors for different fingerings, the characteristics analyzed for this study are generally compared fingering by fingering.

After a description of the experimental setup, the data obtained on the quasi static part of the notes are first compared. Then, the time characteristic of the attack transients, both for the supply pressure and the acoustic oscillations, are studied. The differences observed are interpreted in terms of global characteristics of standard properties of self-sustained oscillator.

\section{Acquisition of data}

\section{$2.1 \quad$ Experimental protocol}

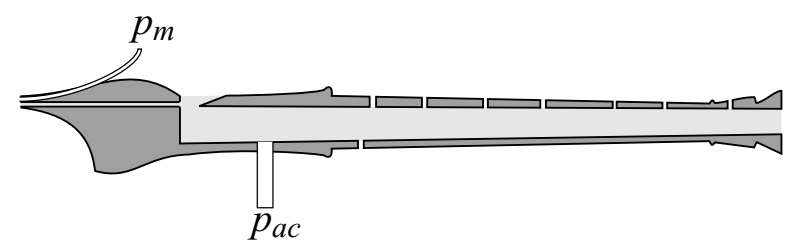

Figure 1: Sketch of the modified recorders that enable the measurement of the acoustic pressure inside the bore $\left(p_{a c}\right)$ and the pressure inside the mouth $\left(p_{m}\right)$.

Two pressure sensors are mounted on four Aesthé recorders, made by the flute maker Jean-Luc Boudreau (figure 1). A bass (from F3 $(175 \mathrm{~Hz})$ to D6 $(1175 \mathrm{~Hz})$ ), an alto (from F4 $(350 \mathrm{~Hz})$ to D7 $(2350 \mathrm{~Hz})$ ), a soprano (from C5 $(523 \mathrm{~Hz})$ to A7 $(3520 \mathrm{~Hz}))$ and a sopranino recorder (from F5 $(698 \mathrm{~Hz})$ to D8 $(4700 \mathrm{~Hz})$ ). The instruments used in this study are those used by Blanc [13]. He uses the recorder geometries parameters to interpret some characteristics of sound and control during steady part of notes played by a musician. Here, the first sensor is a B\&K microphone model 4938 mounted through the wall that measures the inner acoustic pressure $p_{a c}$ near the edge. The second sensor is a Honeywell pressure sensor model $176 \mathrm{PC} 14 \mathrm{HG} 1$. It allows to measure the pressure $p_{m}$ within the mouth of the player thanks to a capillary tube passing through the mouthpiece and ending in the mouth (about $10 \mathrm{~cm}$ long and $1 \mathrm{~mm}$ internal diameter). A calibration of this device against B\&K microphone 4938 and Endevco $8507 \mathrm{C}-2$, shows that its response is flat until about $500 \mathrm{~Hz}$. The delay due to the propagation into the capillary tube is correctly estimated from the 
sound velocity in free field conditions. This delay, about $0.3 \mathrm{~ms}$ is here neglected. The two signals are sampled at $f_{s}=51.2 \mathrm{kHz}$.

Measurements were made with three players: two experienced recorder players with over ten years of practice and formal training in classical recorder, and a novice player with no specific knowledge of recorders or music. To explore all the types of attack transients used by the players in music, the first experienced player was asked to execute two types of exercises with the four recorders: scales on the full compass of the instrument, repeated for different standard attacks, and several musical extracts well known by the player chosen for their specificity and musical context. Due to fault on the keywork of the modified model of bass recorder, only scales were measured with this instrument. The same tasks were asked of the second experienced player but only the soprano and alto recorders were played. The novice player used only the alto and the soprano recorder, and played repetitions of notes for each fingering, with the task of trying to use different attacks. Only the 16 lowest fingerings were used by the novice player (one octave plus three semitones) because partially closed holes are used for higher notes of the range and the portion of the hole which has to be obstructed is not clearly defined. Experienced players claim to constantly be adjusting this partial opening in order to play the notes in tune.

\subsection{Analysis of measurements}

The segmentation of the data is based on the local minima of the mouth pressure to have one attack transient by segment. If the segment contains several linked notes, only the first is kept, based on frequency analysis. This segmentation allows to obtain the associated supply pressure and acoustic pressure (fig. 2) of: 172 occurrences for the bass, 1072 occurrences for the alto, 1150 occurrences for the soprano and 407 occurrences for the sopranino recorder, with the first experienced player, 515 occurrences for the soprano and 390 occurrences for the alto recorders for the second experienced player, and 300 occurrences for the alto and 290 occurrences for the soprano recorder with the novice player.

For a global comparison of these 4300 occurrences, it is necessary to automatically extract some information with algorithms. A lot of characteristics were studied, but only the ones that gave interesting information are presented here. The relationship between the parameters can't directly be observed. As a matter of fact, the different fingerings used to play the different notes of a chromatic scale on a recorder modify the instrument and its proprieties such as the peaks of admittance, their quality factors, the quasi-static performance as function of the mouth pressure, etc. The parameters of the attack transients should therefore be studied by taking into account the recorder and the fingerings used. The fingering is estimated a posteriori from the pitch of the notes. The pitch is estimated as the fundamental frequency $f_{1}$ of the sound, by the Yin algorithm developed by Cheveigné [14]. The number of occurrences for each semitone is different for each player. The novice player played around ten repetitions for each of the 16 lowest semitones. The experienced players played between 20 and 100 repetitions for the most of semitones but some semitones are few 


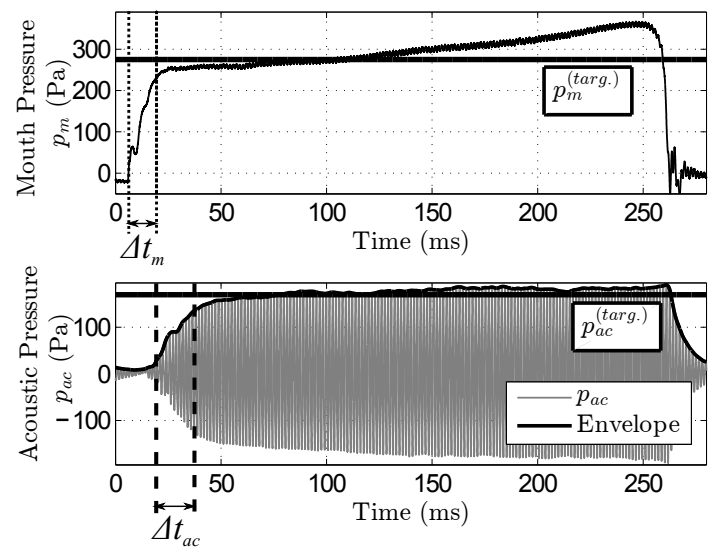

Figure 2: Example of an $E_{5}(659 \mathrm{~Hz})$ played by the first experienced player on the soprano recorder.

or not played due to the tonality of the musical tasks (for example the $C \sharp$ and $G \sharp$ ). The statistical considerations by semitone presented later are led only on semitones with more than five occurrences.

\section{Quasi-stationary part of the note}

\subsection{Supply the instrument}

The target mouth pressure $p_{m}^{(\text {targ. })}$ is a stationary parameter but it participates in the characterization of the attack transient. This characteristic is well defined when the player uses a rectangular time shape of the mouth pressure with a well-defined plateau value. But in general cases, it is harder to define (fig. 2). In this study, the target pressure $p_{m}^{\text {(targ.) }}$ is defined as the average of the mouth pressure, over twenty periods of the fundamental frequency, after the end of the growth. This end of growth is defined as the first instant when the time derivative of the mouth pressure is lower than $10 \%$ of its maximum value, after having reached this maximum. The key point of this algorithm is that it is not sensitive to the evolution of the mouth pressure during the sustain of the note related to the musical task. However, it may provide an erroneous value when the mouth pressure displays non-regular slope during the transient, for example in the case of an overshoot. The statistical analysis chose in this study allows to exclude these extremes values.

The target mouth pressure can be associated with the target dimensionless jet velocity $\theta^{\text {(targ. })}=\frac{U_{j}}{W f_{1}}$ which is a parameter previously used to characterize the behaviors of flute-like instruments in a stationary response $[19,13,8]$, with $W$ the length between the end of the channel and the edge of the recorder and the Bernoulli jet velocity $U_{j}=\sqrt{2 p_{m}^{(\text {targ. })} / \rho}$, with $\rho$ the density of air. The lengths $W$ have been measured by Blanc [13] for the recorders used 
in this study.

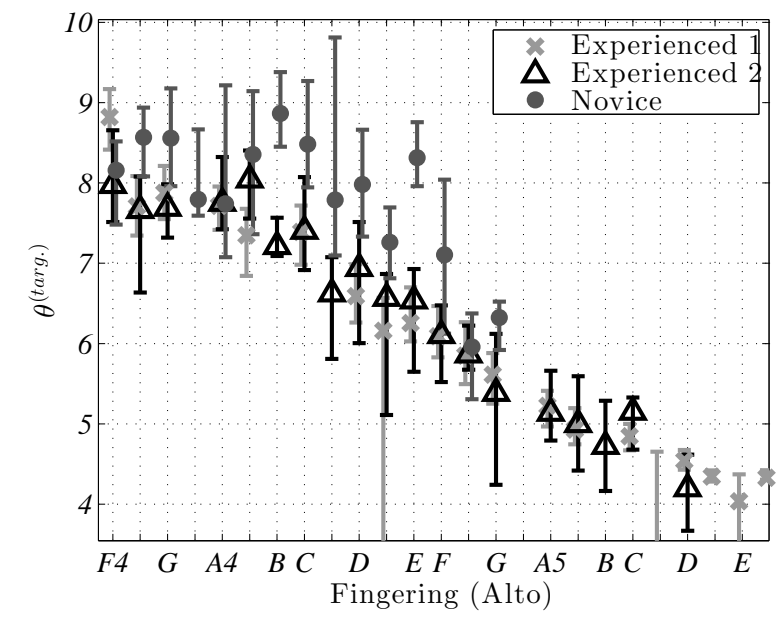

(a) Alto

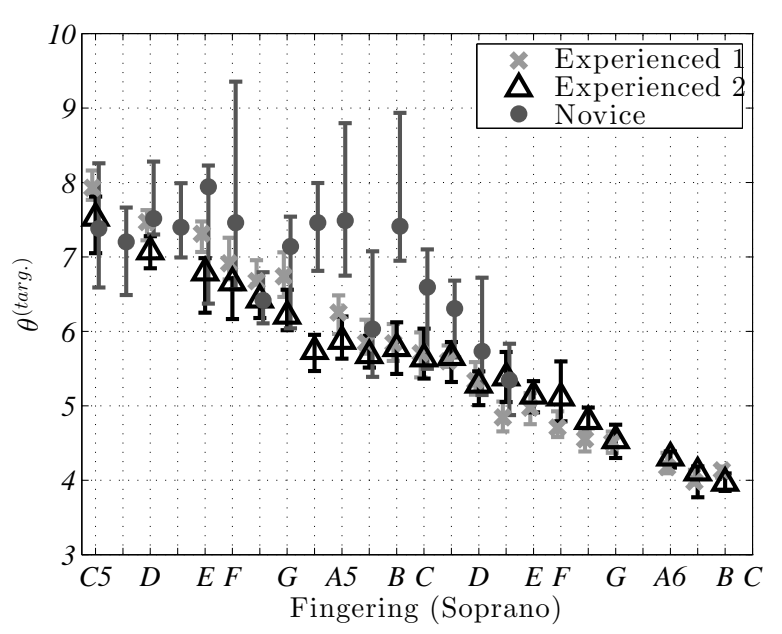

(b) Soprano

Figure 3: Distributions of the dimensionless velocities $\theta^{\text {(targ.) }}$ used by the three players with each fingering of (a) the alto and (b) the soprano recorders. The median values for each fingering are indicated by the markers and the vertical lines represent the IQR (second and third quartiles in the distributions).

The distribution of the values obtained for each fingering of the alto and soprano recorders for the three players are summarized on figure 3. The repartitions of the data by fingering are quantified by the median values the interquartile ranges (IQR), which are the difference between the upper and lower quartiles of each distribution. This distance, not necessarily symmetrical around the median value, includes $50 \%$ of the data. These statistical characteristics minimize the influence of erroneous extreme values and are used in the figures $5,6,7,10,11,14$ and 15 .

The two experienced players used a very small range of dimensionless velocity $\theta^{\text {(targ. }}$ for a given fingering and the values are very close for these two musicians. Overall, the novice player used higher values of $\theta^{(\operatorname{targ} .)}$ with a wider range. In spite of these differences the distributions are not clearly separated and the median values mainly follow the same evolution with the fingering for the three players. These observations suggest that the instruments partly impose the quasi-static supplied pressure for a given fingering. This limitation can partly be explained by the change of regime when the recorder is over-blown [8]: if the player uses a supply pressure that is too high, the recorder plays on the upper octave and the player adapts the mouth pressure. The range of velocity avoiding this over-blowing is very limited on the bottom of the range of the recorders for the four lowest fingerings. This may explain why the distribution of the jet velocity used by the novice player is closer 
to the values used by the experienced players in this range for the two recorders studied (fig. 3).

Blanc [13] observed with an experienced player that, for chromatic scales, the dimensionless velocity $\theta^{\text {(targ. })}$ decreases linearly with the semitones $\left(12 \log _{2}\left(f_{1} / f_{\text {ref }}\right)\right)$. And all the recorders of the family follow the same linear dependency(fig. 4b). The same type of behavior is observed in this experiment when considering the median values for the notes played by the experienced players (fig. 4a).

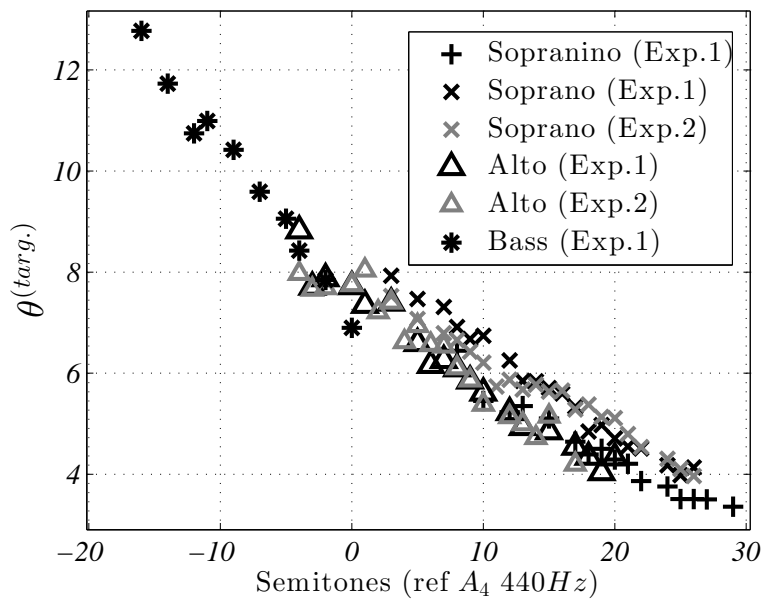

(a) Medians of the dimensionless velocity $\theta^{\text {(targ.) }}$ for the four recorders used by the two experienced players (player 1 in black and player 2 in gray).

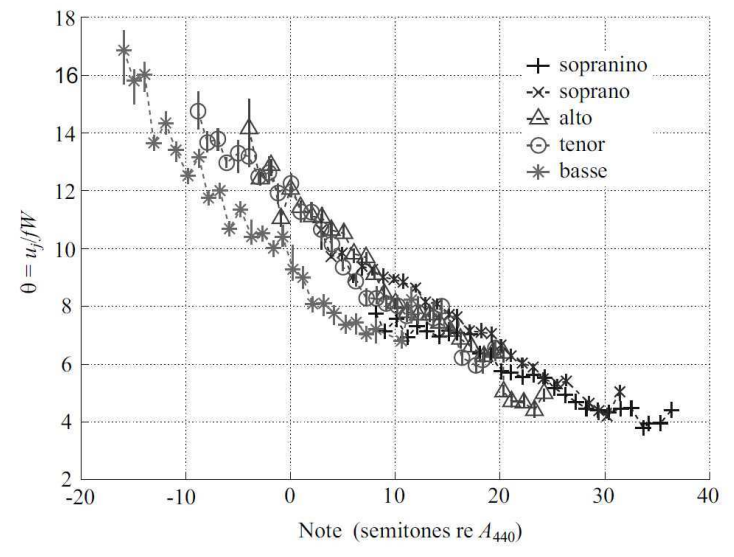

(b) Extract from Blanc [13]:Dimensionless velocity $\theta^{\text {(targ. })}$ as a function of the note played for the five recorders.

Figure 4: Comparison of dimensionless velocity as a function of the note played for this study and the study of Blanc [13].

The global behavior is the same but the slopes are different between the two studies (fig. 4). As the recorders used were the same, this difference can be due to the different performances of the musicians. The difference between the two studies is larger than the small one which can be observed between the two experienced players of this study (fig. 3 and fig. 4). The musical experiences of the three players observed being similar, the difference between the two studies should have another origin. It could be related to the algorithm used by Blanc to determine the target mouth pressure which is not described. The mouth pressure used by the musicians generally increases during the sustain of the note (fig.2). If, in the study of Blanc, the target pressure is estimated from the mean value of the mouth pressure during the duration of the entire note, the values obtained by Blanc should globally be higher than the ones obtained by applying the algorithm based on the derivative used in this study. 


\subsection{Quasi-static sound properties}

Due to the similarity of the observations between the two recorders (alto and soprano), only the aspects of the steady sound of the soprano recorder are discussed here. The following observations are also valid for the alto recorder.

The small range of jet velocities used by the experienced player compared to the novice one (fig. 3) should be associated with desired limitations of acoustical characteristics. The aim of this section is to identify these characteristics. The dimensionless velocity $\theta$ has already been identified to influence the amplitude, the pitch accuracy and the timbre of the sound $[13,8]$. The acoustic pressure $p_{a c}$ is measured inside the resonator. Due to the modal resonance, the dynamic and the spectrum of the radiated sound can't be evaluated from the signal measured without some approximations. In this study, the goal is to compare the sound produced by the players fingering by fingering. For this comparison, the acoustic pressure measured into the resonator (sec.2.1), which stays valid for this comparison, is chosen to study the effect of the control on the sound.

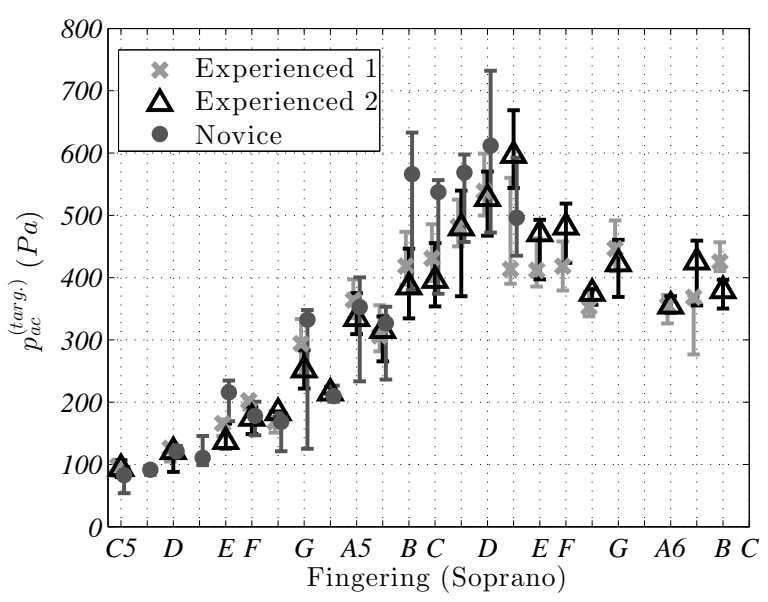

Figure 5: Median values and IQR (sec.3.1) of the target amplitudes of the acoustic pressure oscillation (fig.2), obtained by the three players for the fingerings of the soprano recorder.

The amplitude of the notes produced by the players can be compared through the target amplitude of the acoustic pressure oscillation. The envelope of the acoustic oscillation is estimated from the detection of the local maxima of the absolute value of the acoustic pressure, which are separated at least by $1 /\left(2 f_{1}\right)$. The envelope does not show the overshoot or oscillation, so compared to the mouth pressure, the target amplitude is easier to define. An algorithm based on the time derivative of the envelope is chosen to avoid problems related to the temporal evolution of the amplitude in relation to the musical context such as crescendo, accent, etc. The difference observed between the amplitude produced by the players (fig. 5) is much less pronounced than the one observed in the dimensionless velocity 
(fig. 3b). The accuracy of the control of the experienced player does not seem associated with the amplitudes of the sound.

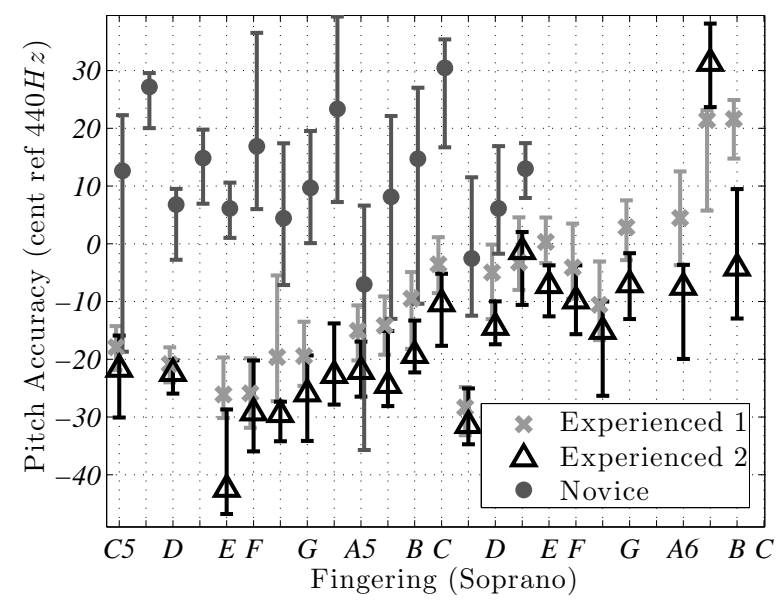

Figure 6: Median values and IQR (sec.3.1) of the pitch accuracy estimated by the difference from the equal temperament scale (ref 440Hz), obtained by the three players for the fingerings of the soprano recorder.

To study pitch accuracy, the fundamental frequencies of the notes are compared in cents to the frequencies of an equal temperament scale with the reference $A_{4}$ at $440 \mathrm{~Hz}$ (fig. 6) $\left(1\right.$ cent $\left.=1200 \log _{2}\left(f_{1} / f_{\text {ref }}\right)\right)$. The two experienced players have very similar profiles of pitch accuracy throughout the range of the recorder and the variation for a given fingering is generally very small ( \pm 5 cents). The novice player clearly played at a higher pitch with a larger scatter. The pitch accuracy appears to be one of the most difficult aspects of the control of steady sound quality on the recorder. The difference observed in pitch accuracy is more marked than the one observed in the dimensionless velocity (fig. 3b). It seems to indicate that the pitch accuracy is not only controlled by the mouth pressure, but also by other types of control such as the fingers.

Both experienced players play too flat at the low end of the range (fig.6). This strange pattern seems to indicate a compromise between the pitch accuracy and the timbre imposed by the instrument. The spectral centroid, already used to characterize the sound of the recorder [13], does not show a clear difference between experienced and novice players. At the low end of the range, it seems that the experienced players want to stay far from the threshold of regime change, which may not have been well adjusted in the recorders used. This could be another explication of this strange pattern. A specific study would be necessary to conclude of the consequences of an inaccurate jet velocity on the quasi-static sound properties.

Having described the observed quasi-static aspects, it is now possible to compare the attack transient of the different players. 


\section{The rising of the supply}

\subsection{Onset times of mouth pressure}

The rise time $\Delta t_{m}$ of the mouth pressure is defined as the time used by the player to go from $10 \%$ to $90 \%$ of the target mouth pressure $p_{m}^{(\text {targ.) }}$. These instants correspond to the instant $t_{m}^{10 \%}$ and $t_{m}^{90 \%}$ respectively, which gives: $\Delta t_{m}=t_{m}^{90 \%}-t_{m}^{10 \%}$. As for the estimation of the target mouth pressure, this algorithm may provide a wrong value when the mouth pressure has a non-regular slope during the transient.

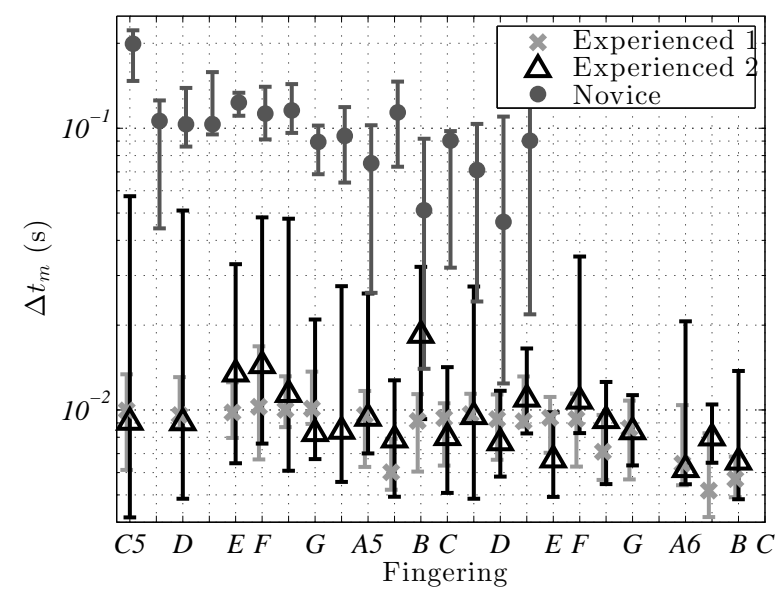

Figure 7: Median values and IQR (sec.3.1) of the rise times $\Delta t_{m}$ of the mouth pressure, obtained by the three players for the fingerings of the soprano recorder.

The comparison of the onset times used by the three players shows that the novice used rise times much longer than the experienced players (fig. 7). There is a difference of more than one order of magnitude between the median values.

\begin{tabular}{|l|c|c|}
\hline Recorder (Player) & Median & IQR \\
\hline Bass (Exp.1) & $15.3 \mathrm{~ms}$ & $21.6 \mathrm{~ms}$ \\
\hline Alto (Exp.1) & $12.6 \mathrm{~ms}$ & $10.4 \mathrm{~ms}$ \\
\hline Alto (Exp.2) & $11.7 \mathrm{~ms}$ & $26.4 \mathrm{~ms}$ \\
\hline Soprano (Exp.1) & $9.4 \mathrm{~ms}$ & $5.3 \mathrm{~ms}$ \\
\hline Soprano (Exp.2) & $8.4 \mathrm{~ms}$ & $13.8 \mathrm{~ms}$ \\
\hline Sopranino (Exp.1) & $12.1 \mathrm{~ms}$ & $8.4 \mathrm{~ms}$ \\
\hline
\end{tabular}

Table 1: Characteristic of the distribution of the rise times of mouth pressure $\Delta t_{m}$, used by the two experienced players for the different recorders. 
The rise times used by the experienced players don't depend on the fingering used and are globally around $10 \mathrm{~ms}$ (fig. 7). It appears that this order of duration of rise times used by the experienced players is the same for all the recorders (table 1).

Different aspects could explain why the experienced players used this order of rise time for the mouth pressure. This value may be the fastest rise possible under physiological limitations. This value may also be fast enough to produce the musical effect desired by the player. Finally, the rise time may be fast enough to produce the fastest possible rise time of the jet velocity. Indeed, as mentioned previously, the physical control parameter of flute-like instrument is the jet velocity $U_{j}$. During a fast attack transient, the evolution of the jet velocity is limited by the inertia induced by the presence of the thin channel between the mouth of the player and the window of the recorder where the jet evolves.

\subsection{Onset times of the jet velocity}

The inertia can be quantified knowing the channel length using the unsteady Bernoulli equation as explained by Verge [15] and Auvray [16]. The resulting central jet velocity $U_{j}$ at the end of the channel is calculated as follows, using the mouth overpressure $p_{m}$, the acoustic pressure at the channel extremity $p_{a c}$ and the channel length $l_{c}$ :

$$
\rho l_{c} \frac{d U_{j}}{d t}+\frac{1}{2} \rho U_{j}^{2}=p_{m}-p_{a c}
$$

where $\rho$ is the air density ${ }^{2}$. Under a given threshold, a faster rise of the mouth pressure doesn't induce a faster rise of the jet velocity. To characterize this effect for the four recorders, the Bernoulli equation (1) is applied on simulated exponential rising of pressure with different characteristic time: $p_{m}(t)=p_{m}^{(\text {targ. })}(1-\exp (-t / \tau))$ (fig. 8). The links between the characteristic time $\tau$ and the rise time $\Delta t_{m}$ is not obvious and is related to the algorithm used to determine the rise time $\Delta t_{m}$. The evolution of the jet velocity being sensitive to the value of the supply pressure, the simulations are calculated for the extreme values used by the players. The rise time of the jet velocity is defined in a similar way to the rise time of the mouth pressure: $\Delta t_{U_{j}}=t_{U_{j}}^{90 \%}-t_{U_{j}}^{10 \%}$, as the time necessary to go from $10 \%$ of the target jet velocity $\left(t_{U_{j}}^{10 \%}\right)$ to $90 \%$ of this value $\left(t_{U_{j}}^{90 \%}\right)$.

For slow transient the jet velocity follows the mouth pressure $\left(\Delta t_{U_{j}}=\Delta t_{m}\right)$. For fast transients, the jet velocity follows the step response of the Bernoulli equation (eq. (1)) which has an exponential growth characterized by the time $2 l_{c} / U_{j}^{(\text {targ. })}$, with $U_{j}^{(\text {targ.) }}$ the target jet velocity. The asymptotic rise time for fast attacks can therefore be estimated analytically by $\Delta t_{U_{j}}=A 2 l_{c} / U_{j}^{(\text {targ. })}$, with $A$ a constant related to the definition of the rise time $\Delta t_{U_{j}}$ used in this study (here $A \approx 1.5$ ). The effect of the channel inertia appears, for mouth pressure

\footnotetext{
${ }^{2}$ During some attack transients, the mouth pressure is lower than the atmospheric pressure during a short time. In this situation, the equation has to be slightly modified to assure the continuity.
} 


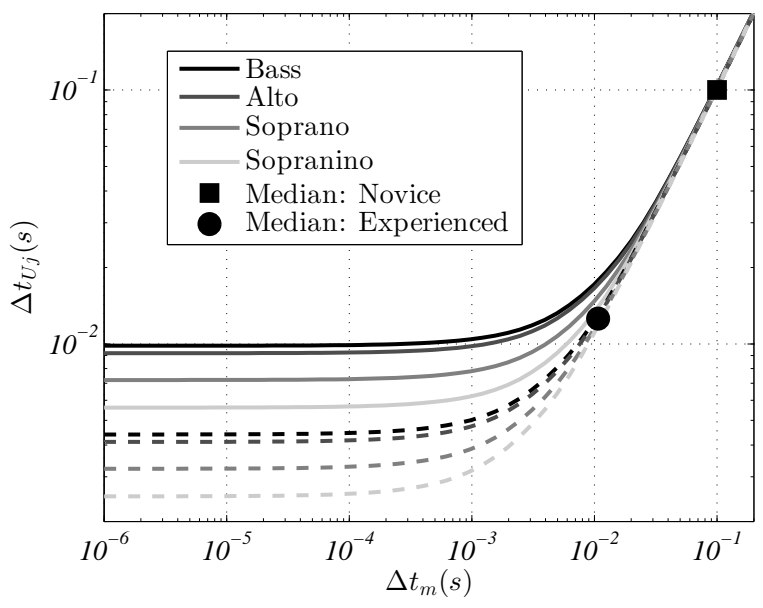

Figure 8: Evolution of the rise time of the jet velocity calculated with the unsteady equation of Bernoulli (1) versus the rise time of the mouth pressure. The bold lines correspond to a target pressure of $p_{m}^{(\text {targ. })}=150 P a$, and the dashed lines to a target pressure of $p_{m}^{(\text {targ. })}=800 P a$. The markers correspond to the median values of rise times obtained by the player (circle: experienced players, square: novice player).

rise times below $10 \mathrm{~ms}$, to produce a saturation of the jet velocity rise time between $11 \mathrm{~ms}$ and $3 \mathrm{~ms}$ according to the recorder channel size and the target mouth pressure (fig. 8).

The Bernoulli equation (eq. (1)) can be applied on the measured mouth pressure to estimate the jet velocity used by the three players. The inertia of the channel attenuates the high frequency during the transient. It generally filters the overshoot and the irregularities in the growth of the mouth pressure. The time shape of the jet velocity is therefore smoother than the time shape of the mouth pressure, which reduces the error in the estimation of the rise time due to non-regular slope of the signal during experimental transient.

As the rise time of the jet velocity is independent of the fingering and recorder used, the values obtained by the three players can be compared (fig. 9). As for the rise of the mouth pressure, the novice player uses rise time an order of magnitude longer than the ones used by the experienced players. The median times used by the players are compared to simulated data of figure 8. It appears that the experienced musicians used the shortest times which allowed them to maintain the control of the rise time of the jet velocity before its limitation by the inertia of the channel (fig. 8). The times used allow them to have a similar evolution of the jet velocity for all the recorders and the target pressures used.

The rise time of the supply appears as the biggest difference of control between the novice and the experienced players. The aim is now to identify the effects of this fast rising of jet velocity on the sound produced. 


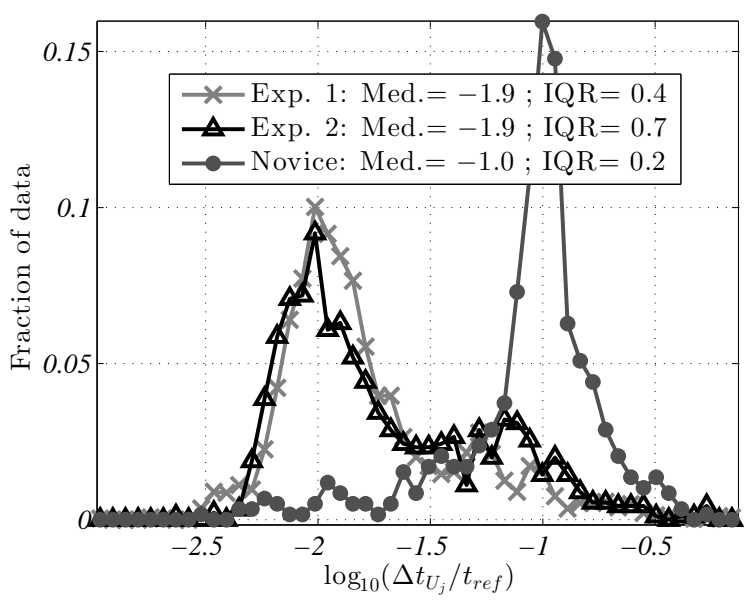

Figure 9: Distribution of the rise times of jet velocity $\Delta t_{U_{j}}$ obtained by the three players on all the recorders used. For each player, the markers correspond to the bar heights of a normalized histogram. Due to the logarithmic scale, the times are normalized by $t_{r e f}=1 \mathrm{~s}$.

\section{Rise and stabilisation of acoustic oscillation}

The rise of acoustic can be decomposed in three steps [10]:

1. The initiation of oscillation: like all auto-oscillating system, a recorder needs a first acoustic perturbation to start its oscillation. This first input of energy is not necessary a harmonic oscillation with a given frequency. In simulation, it is often associated with white noise for accommodation [8]. M.P Verge [11] proposed to assimilate it to the little increase of pressure created by a fast variation of the jet velocity in the confined domain defined by the resonator geometry around the opening where the jet evolved. In the organ community, a lot of denominations are used to characterize the attack transient ("chiff","hiss", etc.). This characteristic could be linked to the initiation mechanism. In some studies this "chiff" is associated with a mouth tone which could be a kind of "edge tone colored by the resonator of the organ" [6, 17].

2. The growth of acoustic pressure: The system accumulates progressively acoustic energy. The efficiency of this accumulation is related to the energy given by the player by blowing and by the capacity of the system to keep this energy at the frequency of the oscillation (quality factor).

3. Saturation of oscillation: the amplitude of the acoustic oscillation stops to increase due to non-linear effects, like, for example, the non-linearity of the acoustic source.

These three steps are not clearly distinct in time domain and their effects are the most often combined to create a specific attack transient. In this study it is proposed to decompose the 
duration of the transient of the acoustic oscillation in two parts:

- the rise time $\Delta t_{a c}$, which is the time taken by the acoustic oscillation to go from $10 \%$ to $90 \%$ of its target amplitude. This time combines the growth and the saturation of the acoustic oscillation. It should exclude the initiation of oscillation.

- the delay, which is the time between the beginning of the growth of jet velocity and the beginning of the growth of acoustic pressure, i.e., the threshold of $10 \%$. The initiation and the beginning of the growth of the oscillation occur during this time.

The control of the musician is first analyzed during the rise time $\Delta t_{a c}$ then during the initial delay.

\section{$5.1 \quad$ Rise time of acoustic pressure}

The rise time of the acoustic pressure is estimated from the envelope of the acoustic oscillation used to estimate the target amplitude $p_{a c}^{(t a r g .)}$ (sec. 3.2), which includes the entire spectrum. The rise time of the acoustic pressure $\Delta t_{a c}$ is the time necessary for the acoustic oscillation to rise from $10 \%$ to $90 \%$ to its target amplitude $p_{a c}^{(\text {targ.) }}$, corresponding to the instant $t_{a c}^{10 \%}$ and $t_{90 \%}^{(a c)}$ respectively, giving: $\Delta t_{a c}=t_{a c}^{90 \%}-t_{a c}^{10 \%}$.

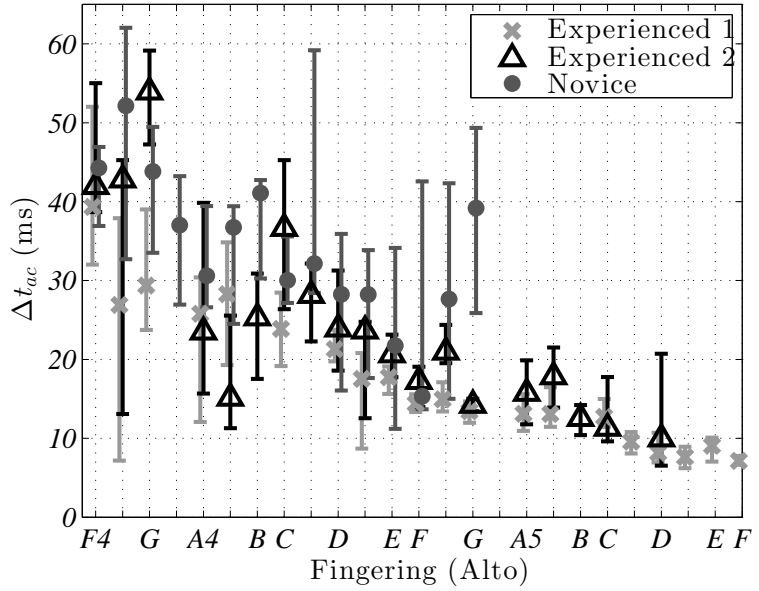

(a) Alto

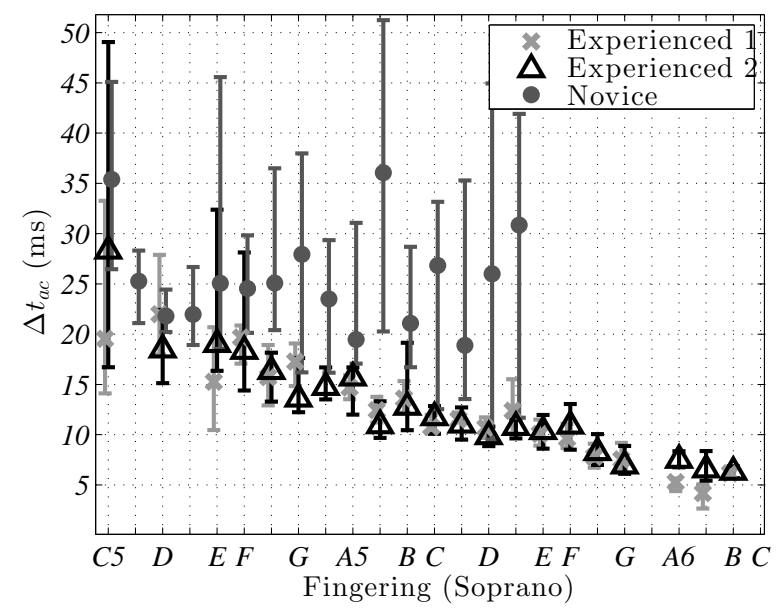

(b) Soprano

Figure 10: Median values and IQR (sec.3.1) of the rise times of the acoustic pressure $\Delta t_{a c}$, obtained by the three players for the fingerings of (a) the alto and (b) the soprano recorders. distributions.

The rise time obtained by this algorithm for the three players is compared for the two recorders in figure 10. Considering the large difference of the jet velocity rise time (fig. 9), the 
three players obtain surprisingly similar rise times for the acoustic oscillation (fig. 10). For low pitches, (under the $E_{5}$ approximately), the median values and the interquartile ranges are very similar for all three players. For higher pitches, the times obtained by the novice player are longer than the ones of the experienced player (fig. 10b). But the difference is still less marked than for the jet velocity rise times. This comparison suggests that the rise times of the acoustic pressure is not a parameter that the musician can finely control. This duration is mostly imposed by the instrument.

For the experienced players, the acoustic rise time is linearly linked to the frequency for the whole range of the instruments. It is therefore possible to define a dimensionless rise time of the acoustic pressure $\Delta t_{a c} f_{1}$. This dimensionless rise time is independent of the fingering, the recorder or the experienced player regarded (table 2).

\begin{tabular}{|l|c|c|}
\hline Recorder (Player) & Median & IQR \\
\hline Bass (Exp.1) & 8.6 & 6.8 \\
\hline Alto (Exp.1) & 11.7 & 3.7 \\
\hline Alto (Exp.2) & 13.3 & 6.8 \\
\hline Soprano (Exp.1) & 12.4 & 3.3 \\
\hline Soprano (Exp.2) & 12.4 & 4.3 \\
\hline Sopranino (Exp.1) & 12.9 & 4 \\
\hline
\end{tabular}

Table 2: Characteristic of the distribution of the dimensionless rise time of acoustic pressure $\Delta t_{a c} f_{1}$, used by the two experienced players for the different recorders.

The times obtained by the novice player follow this behavior for low frequencies but do not go under $25 \mathrm{~ms}$ (fig. 10), obtained around the $E_{5}$. All these observations, added to the fact that the novice player is slower to increase the mouth pressure, seem to indicate that there is a lower limit for the influence of the rise of the jet velocity.

To explain these similarities and differences, it is interesting to observe the time during which the jet velocity continues to increase after the beginning of the acoustic rise $\left(t_{U_{j}}^{90 \%}-t_{a c}^{10 \%}\right)$ on the soprano recorder for which the saturation occurs (fig. 11). Indeed, it has been noted that the acoustic oscillation takes around 12 period to grow and saturates after this time (table 2). For both experienced players, the time during which the jet velocity continues to grow is very small compared to this referenced value of 12 periods. For the novice player, this dimensionless time is in the order of magnitude of 12 periods for the low frequencies, but it is much longer for the high frequencies. The players always used the same range of rise time of mouth pressure (sec. 4.1), but the time response of the instrument is related to the period of the oscillation. For low frequencies, the range of rise time used by the novice player is short enough to grow faster than the acoustic pressure amplitude in the instrument, whereas for high frequencies, it is too slow. It is then possible that, for these high pitches, the velocity continues to evolve after the typical time response of the instrument. The amplitude of the 


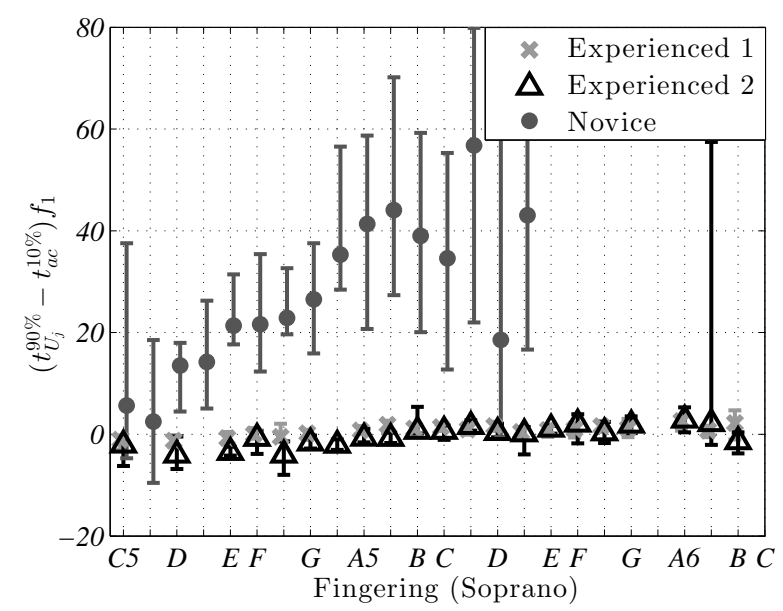

Figure 11: Median values and IQR (sec.3.1) of the dimensionless times during which the jet velocity continues to increase after the beginning of the growth of the acoustic oscillation $\left(\left(t_{U_{j}}^{90 \%}-t_{a c}^{10 \%}\right) f_{1}\right)$, obtained by the three players for the fingerings of the soprano recorder.

acoustic oscillation increases with the jet velocity in a quasi-static way which prolongs the corresponding estimated acoustic rise time. This is maybe why, for high frequencies, longer rise times of the acoustic oscillations are detected by the algorithm.

If the jet velocity reaches its target value shortly after the beginning of the acoustic growth, the acoustic pressure rise time is minimal and proportional to the oscillation period $\left(1 / f_{1}\right)$ of the fundamental. In this case the acoustic oscillation rise time should be independent of the jet velocity rise time. If the jet velocity reaches its target value with a longer time than the typical response time of the instrument, the acoustic oscillation rise time is longer. In this case, the onset time of acoustic pressure is not directly linked to the period of the oscillation and could partially be linked to the rise time of the jet velocity.

This effect in high frequencies excluded, the dimensionless rise time is independent of the fingering and the recorder. It is therefore pertinent to compare the global distribution of the dimensionless rise times of acoustic pressure obtained by the three players (fig. 12). The two experienced players obtain a very similar distribution, and the values produced by the novice player are just slightly more disperse and longer that these ones but they are not clearly distinct. Finally, it appears that, within a certain range, the jet velocity rise time does not influence the acoustic pressure rise time, which is always around 12 periods for the recorders used here.

Considering the definition used for the rise time of the acoustic oscillation, it includes both a part of the growth of the oscillation and its saturation. It appears that the players cannot control these phases of the initial transients. The influence of the fast rise times of mouth pressure or jet velocity, and therefore the aspect controlled by the musician, is 


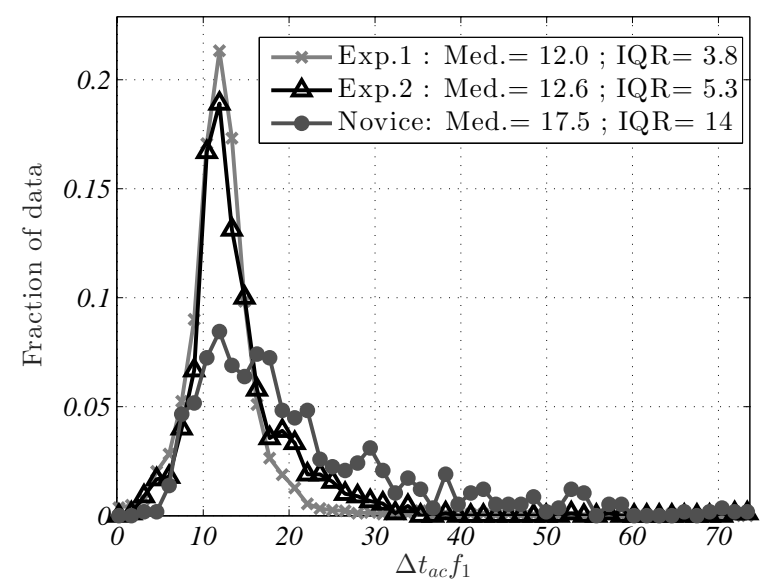

Figure 12: Distribution of the dimensionless acoustic rise times $\Delta t_{a c} f_{1}$ obtained by the three players, including all fingerings and recorders. For each player, the markers correspond to the bar heights of a normalized histogram.

expected during the initiation of the oscillation.

\subsection{Delay between the supply pressure rise and the onset of acoustic oscillation}

The energy needed to initiate the acoustic oscillation is brought to the system between the instant when the player starts to blow and the instant defined as $t_{a c}^{10 \%}$, when the acoustic pressure reaches $10 \%$ of its target value $p_{a c}^{(t a r g .)}$. The quantity of energy initially brought to the system influences its time response. It is then interesting to observe the delay between the growth of the jet velocity and the growth of the acoustic oscillation: $t_{a c}^{10 \%}-t_{U_{j}}^{10 \%}$.

For the three players studied here, this delay appears to be approximately proportional to the fundamental time period of the acoustic oscillation. This relation can be understood by the fact that the delay includes partly the growth of the acoustic oscillation (sec.5.3). The delay normalized by this period appears to be independent of the fingering, which allows a comparison of the global distribution of the dimensionless delays obtained by the three players (fig. 13). It appears that the two experienced players produced the same order of magnitude (between 4 and 7 periods), even if the second one produced slightly longer durations. Most of delays produced by the novice player last between 20 and 30 periods.

It seems that the control of the musicians considerably reduces the time response of the instrument. During this time, two phenomena occur: the injection of the initial energy and the amplification of this small perturbation until the threshold of $10 \%$ of the target amplitude of the acoustic oscillation. 


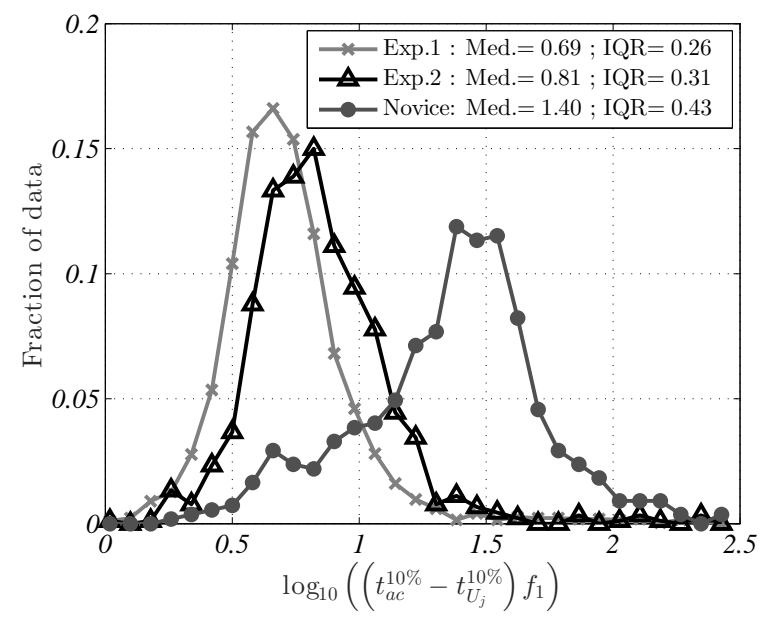

Figure 13: Distribution for the three players, of the dimensionless time-delay between the beginning of the growth of the jet velocity and the growth of the acoustic oscillation : $\left(t_{a c}^{10 \%}-t_{U_{j}}^{10 \%}\right) f_{1}$, including all fingerings and recorders. For each player, the markers correspond to the bar heights of a normalized histogram.

\subsection{Linear response of the instrument}

Like for all systems with a sustained self-oscillation, non-linearity is necessary to saturate the amplitude of the oscillation. In the case of flute, the non-linearities come from both the source created by a jet with a limited width, and by the losses [18]. For small amplitudes of oscillation, such as the beginning of the attack transients, the non-linear effects are negligible and the response of the system can be approximated by a linear response. During the time when this approximation is valid, the amplitude $a_{1}$ of the sinusoidal oscillation at the fundamental frequency $f_{1}$, follows this evolution related to the definition of the gain $G$ of the system:

$$
\begin{aligned}
& a_{1}(t)=a_{1}\left(t-T_{1}\right) G \\
& a_{1}(t)=a_{1}\left(t_{0}\right) G^{\frac{\left(t-t_{0}\right)}{T_{1}}}
\end{aligned}
$$

with $T_{1}=1 / f_{1}$ the fundamental period of the oscillation, and $t_{0}$ the instant when the exponential growth starts. The amplitude $a_{1}$ can be estimated from the weight of the frequency $f_{1}$ in the Fourier transform as described into the next paragraph. In flute-like instruments, the gain depends both on the frequency response of the resonator and on the capacity of the jet and its interaction with the edge to amplify the oscillation at the regarded frequency. If the gain remains constant during the growth of the acoustic oscillation, the amplitude increases exponentially until it reaches the amplitude for which non-linearities take control of the oscillation. Looking for a straight line in the logarithmic representation of the amplitude 
of the acoustic pressure, it is then possible to evaluate the duration of the linear response of the instrument and the gain associated:

$$
G=\exp \left(T_{1} \frac{d\left(\ln \left(a_{1}\right)\right)}{d t}\right)
$$

During some transients it may happen that amplitude of oscillations at different frequencies increase simultaneously, before they decay away as the oscillation stabilizes at the fundamental frequency $[3,20,6]$. These high frequencies have a perceptive importance, but the comparison of transients with and without this phenomenon shows similar development of the fundamental component. These high frequencies are present only during few milliseconds and don't influence the temporal aspects of the transient. They are therefore not taken into account in this study. Equation (4) applies independently on each frequency component; it is necessary to extract the oscillation at the fundamental frequency $f_{1}$ from the acoustic pressure. This extraction is carried using a short-time Fourier transform. The sliding window used is a window of Hann with a time width of $4 / f_{1}$ which is the minimal size which allows the separation of the fundamental frequency from the harmonic resonance. The Fourier transformation, is normalized by the quarter of sample size to identify the maximum of the Fourier transformation around $f_{1}$ with $a_{1}$ the amplitude of the sinusoidal oscillation. An overlap of $80 \%$ between two successive windows is imposed. Equation (4) used on the time evolution of the amplitude $a_{1}$ allows us to evaluate the gains obtained by the three players (fig. 15) for each transient.

The distribution of the gain is very similar for the three players. On the soprano recorder (fig. 14b), the difference between the players is less pronounced than the one between two fingerings. On the alto recorder (fig. 14b), the difference between the novice and the experienced player is more marked but remains in the same order of magnitude as the difference between two fingerings. The values of the gain seem therefore imposed in major part by the instrument. The gain is not very sensitive to the variation of the jet velocity controlled by the players. The difference of gain between the fingerings can be due in part to the frequency response of the resonator, but also to the behavior of the jet, which amplifies more or less a perturbation according to its frequency as calculated for ideal cases by Mattingly and Criminale [21].

Even if the gain seems to be independent of the player, the duration of the linear part and its dependence with the fingering is very different between the experienced and novice players (fig. 15a). For the experienced players, the duration stays around $15 \mathrm{~ms}$ for the high frequencies (higher than $G_{5}$ for both recorder). For low frequencies (lower than $G_{5}$ ), the duration is proportional to the period and of the order of magnitude of 10 periods of the fundamental. It seems that the duration of the linear behavior cannot be shorter than 10 periods and can neither be shorter than $15 \mathrm{~ms}$. For the novice player, the duration is longer and always proportional to the period of the acoustic oscillation (around $\left.17 T_{1}\right)$. As the development of the non-linearities are directly linked to the amplitude of the 


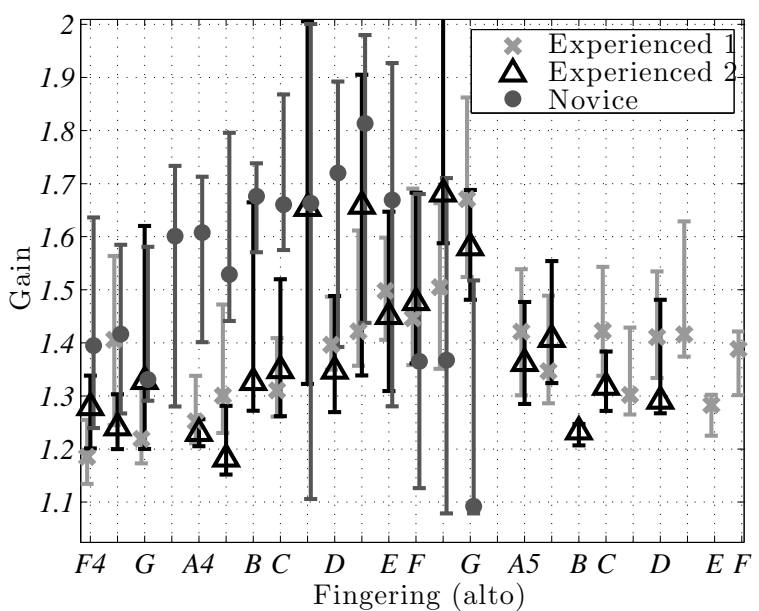

(a) Alto recorder.

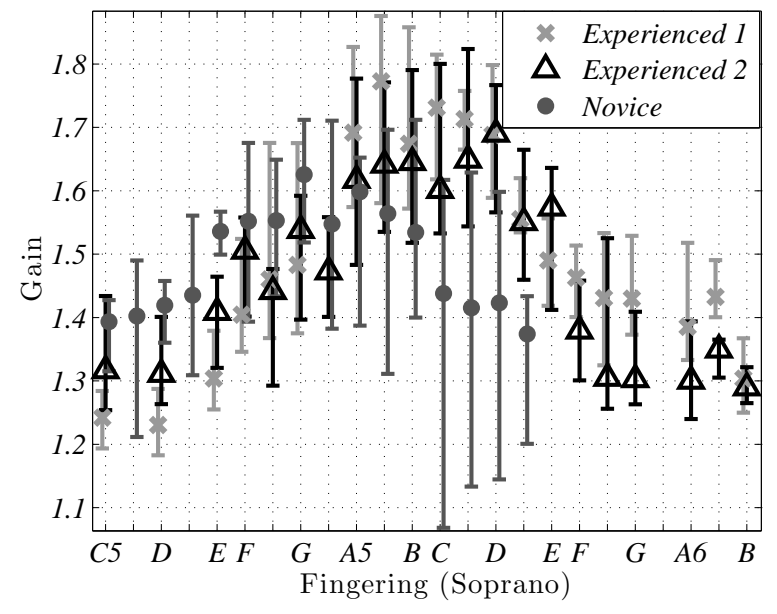

(b) Soprano recorder.

Figure 14: Median values and IQR (sec.3.1) of the gain associated with the linear behavior at the beginning of the attack transients, obtained by the three players for the fingerings of alto (a) and soprano (b) recorders.

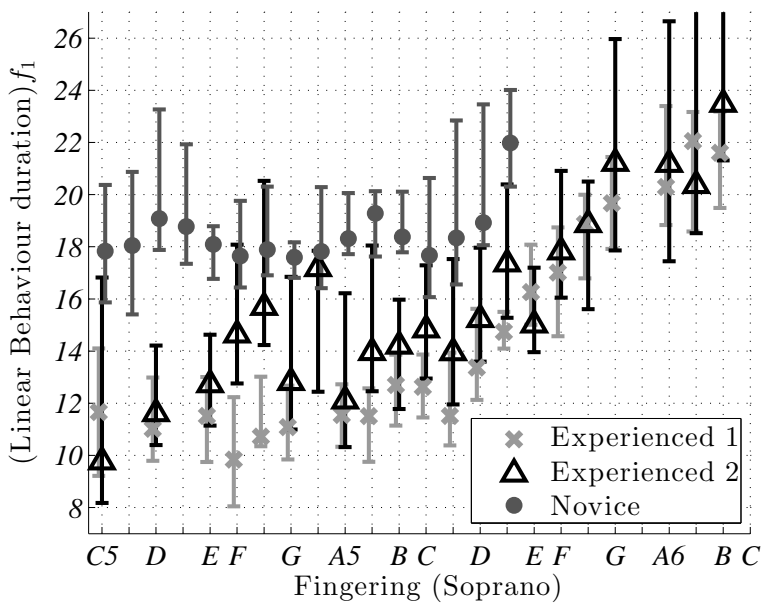

(a) Dimensionless duration of the linear behaviour.

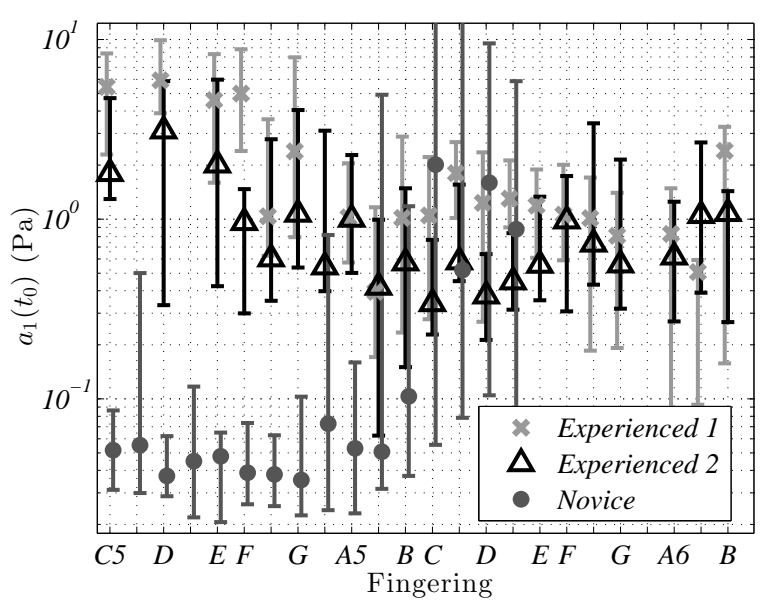

(b) Amplitude $a_{1}$ of the acoustic oscillation at the fundamental frequency when the exponential growth $\operatorname{starts}\left(t_{0}\right)$.

Figure 15: Median values and IQR (sec.3.1) obtained by the three players for the fingerings of the soprano recorder, of (a) the linear behavior duration normalized by the fundamental period and (b) the initial amplitude of the fundamental oscillation $a_{1}\left(t_{0}\right)$. 
acoustic oscillation, the linear approximation ceases to be valid for very similar amplitudes of fundamental frequency for the three players at a given fingering.

The difference of durations between the novice and the experienced players is therefore linked to initial amplitude at the beginning of the exponential growth. The amplitude of the fundamental frequency when the exponential growth starts $a_{1}\left(t_{0}\right)$ is very different for the novice player than for the experienced players (fig. 15b). This difference is especially pronounced for the bottom of the range where they differ by almost two orders of magnitude. This pronounced difference explains why the global duration of the "delay" is so different for the novice player. Indeed, the initial amplitude of the fundamental frequency being weaker, and the gain being the same, the oscillation takes a longer time to rise until the apparition of the non-linearities. Although it was chosen arbitrarily, the threshold of $10 \%$ of the target amplitude is generally attained shortly before the end of the exponential growth. The difference described here occurs before the instant $t_{a c}^{10 \%}$.

The temporal difference between the attacks of the novice and the experienced players seems finally to be concentrated in the initial injection of energy. It is not easy to determine the origin of this injection of energy. It could be associated with an impact of the fingers on the holes at the onset of the note, but this difference of time is also observed in the scale exercises with repetitions of each note during which the musician kept the fingers placed between two attacks. The impact of the tongue on the mouthpiece of the instrument could be another source of energy, but the players said to avoid to touch the mouth piece. This injection of energy seems therefore be only related to the rise of the mouth pressure as

suggested by Verge [11]. A specific study is necessary to characterize this aspect of the control and attempt to understand its origin.

\section{Conclusion and perspectives}

The comparison of attack transients produced by a novice and two experienced players allows us to identify that some aspects of the transient are imposed by the instrument, and others can be controlled by the player. First the target mouth pressure and the equivalent dimensionless jet velocity are observed (sec. 3.1). The behavior of the instrument imposed a limited range of jet velocity for a given fingering. Out of this range, the instrument does not sound or it will change of pipe oscillation mode. The accuracy of the jet velocity used by the experienced players allows them to control the pitch precisely (sec. 3.2).

The rise times of the mouth pressure of the experienced player are more than ten times shorter than the ones of the novice players (sec. 4.1). It appears that they used the shortest times which allowed them to maintain the control of the rise time of the jet velocity before its limitation by the inertia of the channel (sec. 4.2). Regarding mouth pressure, the difficulty of control seems to be associated with the ability to obtain this range of rise time, while keeping the accuracy of the target mouth pressure.

The onset time of the acoustic oscillation is cut in two parts in this study: the birth 
of the oscillation, then its growth and saturation. This second part does not seem to be controlled by the players. The frequency response of the jet associated with the resonator does not seem to be very influenced by the player. The global gain of the system is therefore mainly imposed by the instrument. The non-linear phenomena leading to the saturation of the oscillation are also out of the player's control. The major difference in the temporal evolution of sound, between the novice and the experienced players but also between the two experienced players, is observed during the initiation of the oscillation. The time for the oscillation to reach $10 \%$ of its target amplitude from the beginning of the supply is almost one order of magnitude longer for the novice than for the experienced player, and the distribution of the times obtained by the two players are close but distinct. This duration difference comes from the initial energy input, almost two orders of magnitude lower for the novice player than the experienced one on the bottom of the range.

Finally, the fast growths of mouth pressure used by the experienced players mostly seem to act on this initial input of energy, necessary to initiate the oscillation of the system. To understand the mechanism linking the fast increase of the mouth pressure to the input of energy, it is necessary to identify and characterize this phenomenon by the analysis of its spectral content. As evoked in section 5.3, the recorder being a multi-modal system the oscillations at different frequencies associated with the different regimes of the system can grow simultaneously during the transient. In some transients observed with the three players, the second regime increases initially faster than the first one before it disappears. During a short time, the second regime is dominant in these transients. This perceptible phenomenon has been already observed in organ pipe transients. A quick analysis shows similar proportion of notes presenting this phenomenon for experienced and novice players. The identification of the different aspects, both on the control and on the instruments which promote this phenomenon could help to understand its origin and how the players or the instrument makers can control it. The complexity of the phenomena involved into the development of these components necessitates a specific study concentrates on the time evolution of the spectral content during the attack transient.

\section{References}

[1] D. Deutsch, "Psychology of Music" Elsevier, chap.iv, p.30, 2013.

[2] A. J. M. Houtsma, T. D. Rossing, and W. M. Wagenaars, " Auditory demonstrations on compact disc ", The Journal of the Acoustical Society of America, vol. 83, no.S1, p. S58-S58, May 1988.

[3] J. Keeler, "The attack transients of some organ pipes," IEEE Transactions on Audio and Electroacoustics, vol. 20, pp. 378-391, Dec. 1972. 
[4] A. W. Nolle and T. L. Finch, "Starting transients of flue organ pipes in relation to pressure rise time," The Journal of the Acoustical Society of America, vol. 91, pp. 21902202, Apr. 1992.

[5] M.-P. Verge, Aeroacoustics of confined jets : with applications to the physical modeling of recorder-like instruments. PhD thesis, Technische Universiteit Eindhoven, 1995.

[6] M. Castellengo, "Acoustical analysis of initial transients in flute like instruments," Acta Acustica united with Acustica, vol. 85, no. 3, pp. 387-400, 1999.

[7] J. W. Coltman, "Jet drive mechanisms in edge tones and organ pipes," The Journal of the Acoustical Society of America, vol. 60, no. 3, pp. 725-733, 1976.

[8] R. Auvray, B. Fabre, and P.-Y. Lagrée, "Regime change and oscillation thresholds in recorder-like instruments," The Journal of the Acoustical Society of America, vol. 131, pp. 1574-1585, Feb. 2012.

[9] S. Terrien, R. Blandin, C. Vergez, and B. Fabre, "Regime Change Thresholds in Recorder-Like Instruments: Influence of the Mouth Pressure Dynamics," Acta Acustica united with Acustica, vol. 101, pp. 300-316, Mar. 2015.

[10] N. H. Fletcher, "Transients in the Speech of Organ Flue Pipes - A Theoretical Study," Acta Acustica united with Acustica, vol. 34, pp. 224-233, Feb. 1976.

[11] M. P. Verge, R. Caussé, B. Fabre, A. Hirschberg, A. P. J. Wijnands, and A. van Steenbergen, "Jet oscillations and jet drive in recorder-like instruments," Acta acustica, vol. 2, no. 5, pp. 403-419, 1994.

[12] A. Ernoult, "Experimental Study of Attack transients in flute-like instruments," in International Symposium on Musical Acoustics, (Le Mans), pp. 177-182, 2014.

[13] F. Blanc, B. Fabre, N. Montgermont, P. De La Cuadra, and A. Almeida, "Scaling of Flute-Like Instruments: An Analysis from the Point Of View of the Hydrodynamic Instability of the Jet," Acta Acustica united with Acustica, vol. 96, pp. 642-653, July 2010 .

[14] A. de Cheveigné and H. Kawahara, "YIN, a fundamental frequency estimator for speech and music," The Journal of the Acoustical Society of America, vol. 111, no. 4, p. 1917, 2002.

[15] M. P. Verge, B. Fabre, W. E. A. Mahu, A. Hirschberg, R. R. v. Hassel, A. P. J. Wijnands, J. J. d. Vries, and C. J. Hogendoorn, "Jet formation and jet velocity fluctuations in a flue organ pipe," The Journal of the Acoustical Society of America, vol. 95, pp. 1119-1132, Feb. 1994. 
[16] R. Auvray, B. Fabre, P.-Y. Lagrée, S. Terrien, C. Vergez, and others, "Influence of the fluctuations of the control pressure on the sound production in flute-like instruments," Acoustics 2012 Nantes, 2012.

[17] A. Miklós and J. Angster, "Properties of the Sound of Flue Organ Pipes," Acta Acustica united with Acustica, vol. 86, pp. 611-622, July 2000.

[18] B. Fabre and A. Hirschberg, "Physical modeling of flue instruments: a review of lumped models," Acta Acustica united with Acustica, vol. 86, no. 4, pp. 599-610, 2000.

[19] M.-P. Verge, A. Hirschberg, and R. Caussé, "Sound production in recorderlike instruments. I. Dimensionless amplitude of the internal acoustic field," The Journal of the Acoustical Society of America, vol. 101, no. 5, pp. 2914-2924, 1997.

[20] M.-P. Verge, A. Hirschberg, and R. Caussé, "Sound production in recorderlike instruments. II. A simulation model," The Journal of the Acoustical Society of America, vol. 101, no. 5, pp. 2925-2939, 1997.

[21] G. E. Mattingly and J. O. Criminale, "Disturbance Characteristics in a Plane Jet," Physics of Fluids (1958-1988), vol. 14, pp. 2258-2264, Nov. 1971. 